\title{
A Century's Breakthrough in Upcycling Lignocellulosic Biomass: Embion Technologies, Hard-tech Spin-off of the EPFL, Disrupts Nutrition Innovation with Sophisticated, Complex-prebiotics for Immunity
}

\author{
Gina Noh, Cora De Gol, and Sviatlana Siankevich*
}

\begin{abstract}
Embion Technologies SA is a hard-tech spin-off of the EPFL, with a disruptive and novel platform technology that aims to enable the global transition to zero waste via the circular bioeconomy. Embion's initial focus is on transforming low-value food and agricultural industry byproduct streams to affordable next-generation prebiotics nutrition for human and animal microbiome. We demonstrate here that the company's proprietary technology is simple and flexible and can be applied to a wide variety of feedstocks to extract tailored products with specific fingerprints. Embion's technology unlocks the natural hidden value of fibers, fats, proteins, minerals, and polyphenols within these byproduct streams to bring different functional food ingredients to the food, feed, and beverage market.
\end{abstract}

Keywords: Bioactives $\cdot$ Immunity $\cdot$ Lignocellulosic biomass $\cdot$ Prebiotics

Gina Noh is Catalyst Process Technology Specialist at Embion Technologies. She is a chemical engineering professional with $10+$ years of R\&D experience in both industry and academia. She has previously worked at General Electric \& ExxonMobil on several research projects. She has a BS and MS in chemical engineering from the MIT and a PhD in chemical engineering from the University of California. Her expertise is focused on synthesis and catalyst development for hydroisomerization and methanol synthesis.

Cora De Gol is R\&D Specialist and Production Manager at Embion Technologies. She has expertise in carbohydrate chemistry and hydrolysis processes acquired in several large projects at Nestlé, where she worked with enzymes or catalysts on multiple types of biomasses. At Embion Technologies she is responsible for product development and oversees prebiotic activity testing of developed products. She holds a MSc in chemistry and bioengineering with a specialization in sustainable food processing as well as a BS in chemistry and chemical Engineering from ETHZ and EPFL.

Sviatlana Siankevich is CTO of Embion Technologies. She brings $10+$ years of technological expertise in biomass valorisation, having worked with technologies ranging from enzymes and fermentation to chemical synthesis and catalytic transformations. She has a strong academic track record and has released 4 patents \& patent applications. She has a diploma in ecology from the International Sakharov Environmental Institute in Minsk (Belarus), a MSc in chemical engineering from university of Patras (Greece) and a PhD in chemistry from EPFL.

*Correspondence: Dr. S. Siankevich, E-mail: ssiankevich@embiontech.com, Embion Technologies SA, Chemin de la Dent d'Oche 1A, EPFL Innovation Park (Building L), $\mathrm{CH}-1024$ Ecublens, Switzerland

\section{Embion Technologies - Background}

Embion Technologies S.A. is a hard-tech, disruptive spin-off of the Swiss Federal Institute of Technology in Lausanne (EPFL), providing access to next-generation nutrition innovation through a novel platform technology. The patented technology platform is based on the principle of bold simplicity - Embion empowers a scalable and affordable way to meet the globally growing demand for highly functional plant-based nutrition. Embion, 'The Life Cycle Company', supports global development of a bioeconomy. Through its proprietary rapid-prototyping platform, the company enables the upcycling of industrial biomass side-streams into soluble bioactive nutrition for human and animal health.

Embion Technologies was co-founded in 2016 by Georgios Savoglidis, Sviatlana Siankevich, and Georgios Fengos, three scientists trained in chemical engineering at the EPFL. The founding team originally met while studying at the University of Patras in Greece, where they shared a common vision of inventing novel high-tech and agile processes with global benefits for the environment and for humankind. After many years gaining expertise, the best opportunity came when they met again at EPFL.

\subsection{Ensuring a Transition to Zero Waste}

Embion's mission is to catalyze the world's transition to zero waste with their breakthrough process to transform biomass into value-added products for food, feed, and energy. Food supply chain waste represents a significant societal burden with global implications. ${ }^{[1]}$ According to the 2013 Food Wastage Footprint report ${ }^{[2]}$ published by the Food and Agricultural Organization of the United Nations, the huge quantities of food waste generated annually (1.3 billion tons of edible food) represented $30 \%$ of the total land area used for agriculture and accounted for more than 3.3 billion tons of $\mathrm{CO}_{2}$ emitted in 2013 alone. In addition to waste streams of edible food, food and agricultural waste are generated at the pre-consumer level (post-harvest and processing), which represent byproduct streams that are incompatible with human 
consumption (e.g. pomegranate peels and seeds, fruit pomace). [3] Strategies to valorize these food waste and food byproduct streams are therefore of great interest, both commercially and for sustainability.

The Embion process helps reduce the carbon footprint by decreasing the amount of food waste and improving the efficiency of arable land usage. Embion's technology platform helps reduce $\mathrm{CO}_{2}$ emissions through improvements in overall process efficiency, thus reducing energy costs, compared to current industrial processes. Embion's ultimate goal is to enable the transition of many different industries to sustainable production and circular business models. The initial focus is on segments of the food and beverage industry that produce large volumes of side streams to generate bioactive functional-food components (shown in Fig. 1). Embion's first commercial application of their technology platform enables the production of affordable next-generation prebiotics, the nutrition for the microbiome.

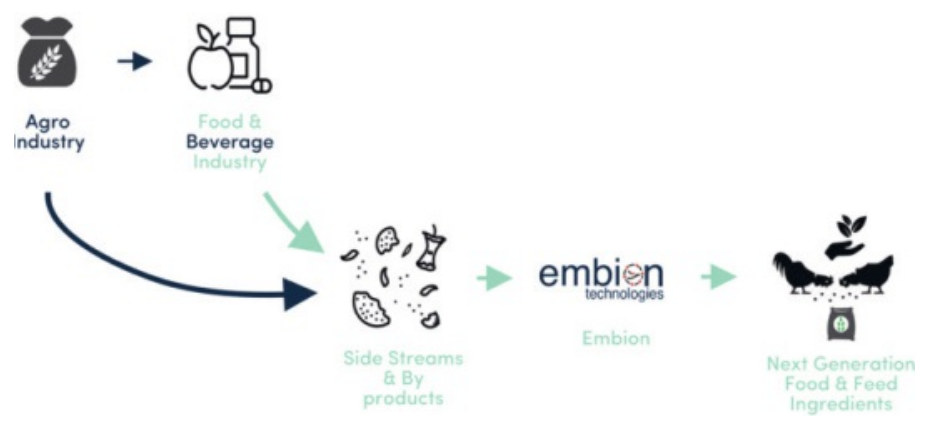

Fig. 1. Embion's place in the circular bioeconomy.

\subsection{The Human Microbiome and Human Health}

The microbiome is composed of the trillions of micro-organisms that inhabit our bodies, for example, living on our skin, in our mouths, and in our gastrointestinal (GI) tracts. ${ }^{[4,5]}$ Estimates suggest that these micro-organisms outnumber human cells by about ten-fold, with the greatest number being located in the distal ileum and the colon. ${ }^{[6]}$ Through millions of years of co-evolution, the host-microbiota interaction is largely mutually symbiotic: the host provides a hospitable and stable environment for the growth of these microbes, while the microbes contribute to physiological processes for their human hosts that were not independently evolved. For example, specific species of bacteria are responsible for the production of short-chain fatty acids (SCFA) via fermentation of nondigestible carbohydrates. ${ }^{[5]}$ SCFA are beneficial to their hosts because of their immunomodulatory effects and their inhibition of common pathogens. ${ }^{[7]}$

Disruptions in the microbiome, referred to as dysbiosis, can result from medication, poor nutrition, aging, pathogens, etc., ${ }^{[8]}$ and can disturb the balance of the microorganisms, resulting in the favored growth of potentially pathogenic organisms. ${ }^{[6]}$ Dysbiosis has been linked to a variety of acute and chronic disorders and diseases, including inflammatory bowel disease, obesity, asthma, and cancer, among many others. ${ }^{[5,6,8]}$ This link between a healthy microbiome and a healthy human host is, in part, the result of the high number of immune cells that reside in the gut, as the intestine is the largest compartment of the human immune system ${ }^{[9]}$ and is continually exposed to a wide range of beneficial and harmful immune stimuli.

Although there are significant variations among the specific microbial composition of individuals, depending on host geographical location, early-life exposures, genetics, and diet, there is a general observation of increased microbiome diversity resulting in improved health. It has been hypothesized that reduced gut microbial diversities, which may be linked to diet high in fats and refined sugars and low in fiber, ${ }^{[10,11]}$ may result in higher rates of chronic disease. ${ }^{[5]}$ Diet therefore can likely greatly influence the functionality and health of the gut microbiome, thereby resulting in improved health for the human host.

\subsection{Prebiotics - The Future of Health}

There are many compounds in food that stimulate the growth or activity of a limited number of colonic bacteria, thereby improving gut health. A prebiotic compound specifically is defined as "a substrate that is selectively utilized by host microorganisms conferring a health benefit."[12] These food components generally share the further characteristics of being nondigestible by the host by resisting gastric acidity and being fermented by intestinal microbiota. ${ }^{[13]}$ Prebiotics are selectively utilized by the microbes in the gut, increasing bacterial growth and improving the functionality of specific bacteria. Either of these factors alone, or both together, can result in health benefits. For example, metabolic products such as SCFA lower intestinal $\mathrm{pH}$, thereby improving mineral absorption by the intestinal wall while also providing an unfavorable environment for pathogens. ${ }^{[8]}$

Inulin (fructooligosaccharide (FOS)) and galactooligosaccharide (GOS), have been widely commercialized as prebiotic dietary products. ${ }^{[14]}$ Inulin and GOS, which are derived from chicory root or Jerusalem artichoke by extraction using a hot water diffusion process or synthesized from sucrose via enzymatic transfructosylation, ${ }^{[15]}$ currently have the largest market share (accounting for $40 \%$ of the prebiotics market in 2015 by revenue). ${ }^{[16]}$ Other oligosaccharides of low molecular weight have shown particular promise as prebiotics because they are reported to be suited to the growth and activity of bifidobacteria and lactobacilli, two major genera of bacteria in the gut. The lower degree of polymerization, compared to polysaccharides, has been linked to increased prebiotic activity.[17] Lactulose, xylooligosaccharide (XOS), arabinoxylan-oligosaccharides (AXOS), and isomaltooligosaccharide (IMO) have specifically been identified as promising candidate prebiotics that are currently in varied stages of development and commercialization. ${ }^{[14,18]}$

XOS \& AXOS are the hydrolytic degradation products of heteroxylans (also referred to as arabinoxylans, AX), which are a major component of the dietary fiber in cereal grains. ${ }^{[19]}$ AXOS has demonstrated a clear bifidogenic effect, and its use has resulted in improved markers of health by a number of different metrics (e.g. potential anticarcinogenic effects, modulation of high serum cholesterol, triglyceride, and glucose levels as a result of poor diet). ${ }^{[19]}$ Although recommended daily intake of XOS is lower than of inulin/FOS (4.2 $\mathrm{g}$ vs $10 \mathrm{~g}),{ }^{, 14]}$ it is currently more expensive to produce. However, XOS/AXOS have shown higher resistance to digestion and more effective bifidogenic activity, ${ }^{[14]}$ suggesting that it is a more efficacious prebiotic oligosaccharide.

Improvement of health outcomes via manipulation of the microbiome applies not only to humans but also to animals. Similar phenomena are at play for the chicken gut microbiome. In commercial broiler production, the European Union ban on antibiotic growth promoters has been associated with increased incidence of intestinal disorders in chickens. For example, since the ban, necrotic enteritis has become a widespread and costly disease in poultry. The potential to manipulate the intestinal microbiota for chickens could lead to improved health outlooks and animal productivity. ${ }^{[20]}$ Regulating dysbiosis requires combined prebiotic and probiotic intervention, especially at the early stages of life immediately following hatching, in order to colonize the chickens with healthy bacteria. In ovo interventions have led to improved tolerance to environmental stresses at grower houses and to optimized gut health and bird performance. ${ }^{[20]}$ AXOS specifically has shown promise as a prebiotic additive for broilers, with positive effects on net utilization of dietary energy and bird performance. [21] 
Many of the polysaccharide precursors for these prebiotic compounds are contained in lignocellulosic food and agricultural byproduct streams. The current methods of obtaining oligosaccharides are enzymatic, microbial, chemical, and autohydrolytic. Traditional chemical and autohydrolytic processes have disadvantages of producing undesired and potentially toxic byproducts and low control over degree-of-polymerization. In addition, these processes use organic solvents and/or mineral acids/bases, which are difficult to handle and require special disposal. Enzymatic and microbial processes are more environmentally friendly, operating at mild temperatures without use of organic solvents, but the enzymes capable of these substrate-specific reactions can be expensive if commercially available or must be produced. ${ }^{[14]}$

\section{Embion's Approach: A Flexible Technology Platform}

Embion has developed innovative, eco-friendly processes for biomass valorization that are inspired by ionic liquid-catalyzed chemistries. ${ }^{[22]}$ The Embion catalysts are efficient at targeted depolymerization of the polymeric macronutrients comprising lignocellulosic biomass. Specifically, fibers, proteins, starches, fats, etc., can be selectively hydrolyzed to produce a wide variety of desired products (as shown schematically in Fig. 2). The novelty of Embion's processes is in their unique capability to efficiently extract functional ingredients from low value resources, typically considered waste streams of food and agricultural industries, such as fruit pomace, stems of plants (e.g. corn, tomato, grass, and others), wood trimmings, and grain byproducts.

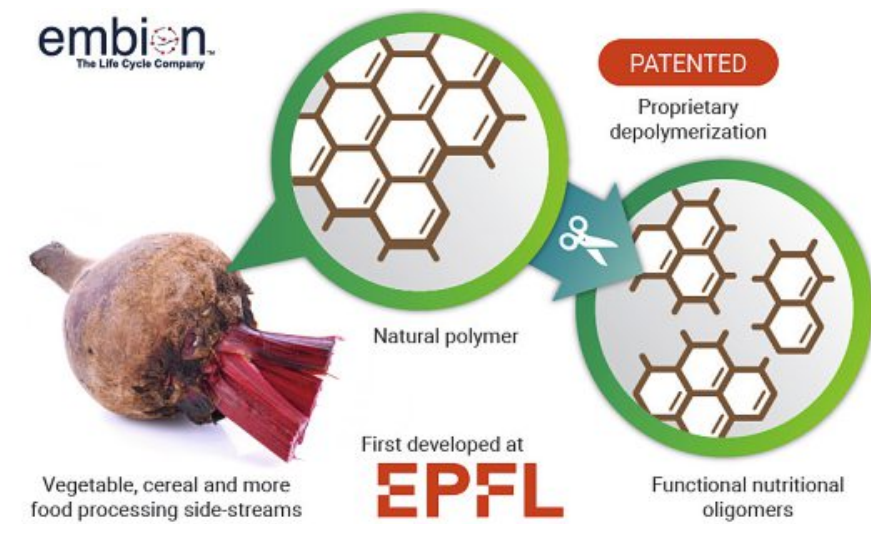

Fig. 2. Representation of Embion's proprietary technology.

The design of the catalyst was based on ionic liquids, which were first discovered in $1914{ }^{[23]}$ and observed to dissolve cellulose nearly a century later in 2002.[24,25] Ionic liquids are composed of large organic ions that are characterized by a high degree of conformational flexibility. ${ }^{[26]}$ Their anions and cations can be changed independently, resulting in tunability of their composition and therefore in their functional performance. By carefully selecting the ions that compose an ionic liquid, it is possible to use the compound as both solvent and catalyst for lignocellulosic biomass conversion. ${ }^{[25]}$ However, in spite of their high efficacy, their industrial use has largely been precluded by the challenges in separation from residual biomass or products.

Embion's novel catalyst technology leverages the advantages of ionic liquids with those of solid catalysts by integrating them into a solid-state ionic polymer (Fig. 3). This approach produces a solid catalyst, which can be readily separated, and retains the ionic features of ionic liquids while improving their thermal stability. Embion catalysts are based on a flexible and modular structure, agnostic to biomass feedstock type, that can be easily tailored to fine-tune product composition. In particular, through structural changes and functionalization of the catalyst, the composition of the products extracted from the biomass can be controlled. The

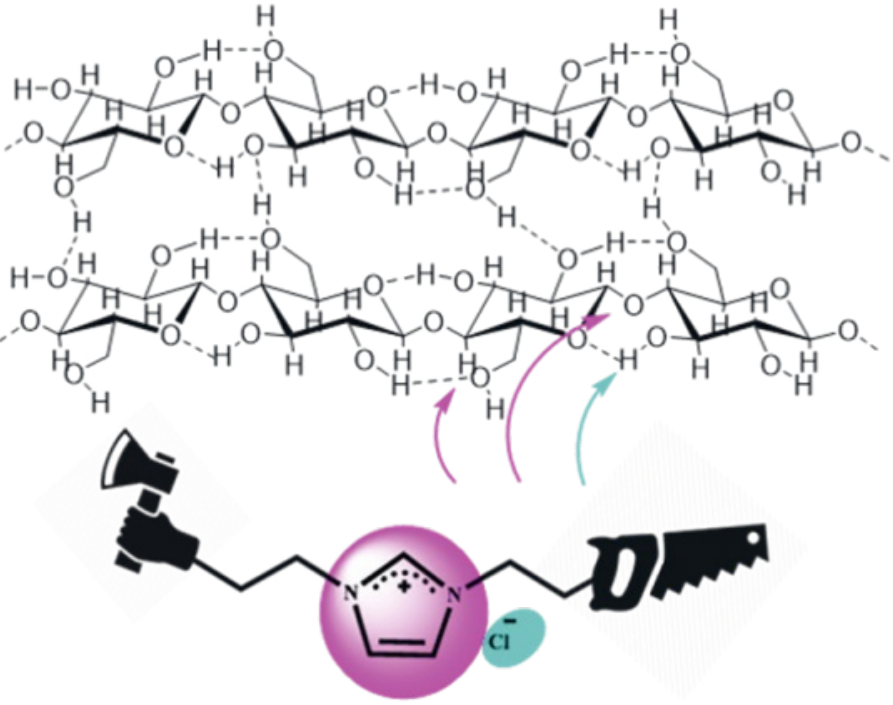

Fig. 3. Schematic representation of the Embion catalyst and how its cation (magenta) and anion (cyan) interact with biomass for selective hydrolysis.

Embion process for extraction of plant-based compounds operates in water under mild conditions and requires no additives or buffers.

Next, we will discuss specific aspects of Embion's technology platform that make it amenable for diverse applications. The initial focus has been on producing oligosaccharides with prebiotic functionality from lignocellulosic food and agricultural byproduct streams (Section 3). Thus, the focus of this section is on conversion of carbohydrate-based macromolecules.

\subsection{Efficient at Converting a Wide Array of Feedstocks}

Each Embion catalyst can be used to convert a wide array of biomass feedstocks, derived from the waste of food and agricultural industries. Table 1 shows the conversion of corn stover, corn cobs, and brewer's spent grain, using the same catalyst. These feedstocks differ in their macromolecular composition, with this specific corn cob feedstock assessed to be $68 \%$ carbohydrate and corn stover $59 \%$. Brewers' spent grain, a waste product from beer brewing, is composed of 30-45\% carbohydrates but also contains roughly $20 \%$ proteins. We note that the specific macronutrient composition of each feedstock depends on the specific source for that feedstock, as a result of differences in cultivars and agricultural/climate conditions, as well as the process from which it was derived. Notably, the final product of the corn stover contained $84 \%$ of the XOS contained in the feedstock and only $55 \%$ of the contained glucans, demonstrating that the catalyst was also more selective for xylans compared to glucans.

Embion catalysts have been used to convert a wide variety of biomass feedstocks. These feedstocks vary in composition from inulin, which is a polymer of fructans and composed exclusively of carbohydrates, to olive pomace, the byproducts that remain after olive oil extraction, which has a high lipid content. In all cases, the catalyst is able to convert a high percentage of the available carbohydrates.

Table 1. Carbohydrate conversion for different biomass feedstocks.

\section{Conversion $^{\mathrm{a}}$}

Corn stover

$70 \%$

Corn cob

$50 \%$

Brewer's spent grain

$60 \%$

apercent converted of carbohydrates in the feedstock 


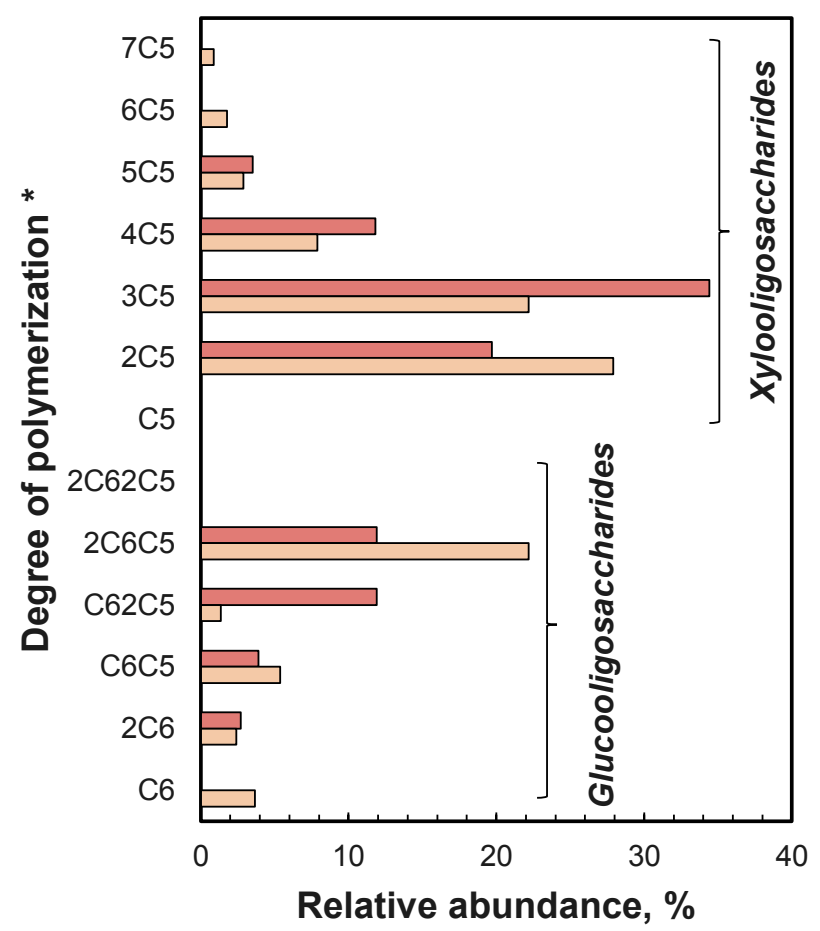

Higher temperature, shorter time; $75 \%$ carbohydrate conv.

Lower temperature, longer time; 50\% carbohydrate conv.

Fig. 4. Degree-of-polymerization profile for products of corn cob hydrolysis using an Embion catalyst at two different conditions (red is higher temperature and shorter time, yellow is lower temperature and longer time). ${ }^{*} \mathrm{C} 5$ and $\mathrm{C} 6$ refer to the pentose and hexose sugar monomers, respectively. The number before $\mathrm{C} 5$ or $\mathrm{C} 6$ corresponds to the number of monomers in the oligosaccharide.

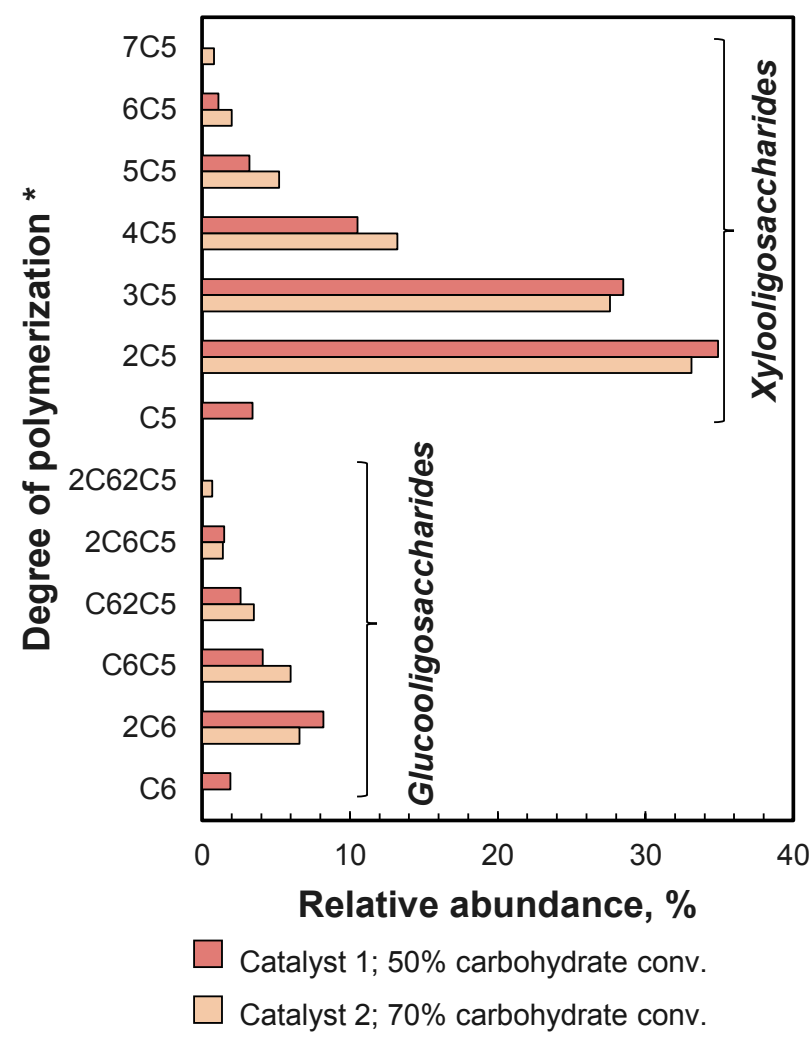

Fig. 5. Degree-of-polymerization profile for products of corn stover hydrolysis using two different Embion catalysts at the same reaction conditions. ${ }^{*} \mathrm{C} 5$ and $\mathrm{C} 6$ refer to the pentose and hexose sugar monomers, respectively. The number before $\mathrm{C} 5$ or $\mathrm{C} 6$ corresponds to the number of monomers in the oligosaccharide.
Table 2. Composition (g/100 g) of corn stover hydrolysis products under the same reaction conditions with different catalysts.

\begin{tabular}{|l|c|c|c|}
\hline & $\begin{array}{c}\text { Total } \\
\text { carbohydrate }\end{array}$ & Glucooligosaccharides & XOS \\
\hline $\begin{array}{l}\text { Corn } \\
\text { stover }\end{array}$ & 59 & 31 & 28 \\
\hline Catalyst 1 & $\begin{array}{c}30 \\
(50 \%)^{*}\end{array}$ & $\begin{array}{c}8 \\
(81 \%)^{*}\end{array}$ & $\begin{array}{c}23 \\
(26 \%)^{*}\end{array}$ \\
\hline Catalyst 2 & $\begin{array}{c}41 \\
(70 \%)^{*}\end{array}$ & $\begin{array}{c}24 \\
(55 \%)^{*}\end{array}$ & $(84 \%)^{*}$ \\
\hline
\end{tabular}

*Value in parentheses indicates the percent converted of the feedstock

Table 3. Composition ( $\mathrm{g} / 100 \mathrm{~g}$ ) of two brewers' spent grain feedstocks of different sources after hydrolysis with $\mathrm{H}_{2} \mathrm{SO}_{4}$.

\begin{tabular}{|l|c|c|}
\hline & Feedstock 1 & Feedstock 2 \\
\hline Total carbohydrate & 39 & 36 \\
\hline Glucose & 11 & 7 \\
\hline Xylose & 20 & 19 \\
\hline Arabinose & 8 & 9 \\
\hline
\end{tabular}

\subsection{Product Fingerprint can be Tuned}

In addition to the ability of these catalysts to selectively convert the carbohydrate fraction of these biomass feedstocks, the specific composition (or fingerprint) of the produced oligosaccharides is crucial to their application as prebiotics. XOS and AXOS with degrees-of-polymerization between 2-10 have shown promising results in terms of their prebiotic activity.[17]

The Embion technology platform can carefully tune the composition of the products by altering reaction time, reaction temperature, and catalyst structure. For example, by changing the conditions of the catalytic reaction, the total oligosaccharide yield and the degree-of-polymerization of these oligosaccharides can be tuned. In Fig. 4, we show the degree-of-polymerization profile of the product oligosaccharides from corn cobs. By changing reaction time and temperature, the product distribution can be shifted to higher degree of polymerization and completely excludes monomeric sugar products.

Changing catalyst composition for the given feedstock (corn stover), while keeping process conditions the same, also shows significant changes in the degree-of-polymerization profile of the product oligosaccharides (Fig. 5). For example, changing the catalyst composition also resulted in the absence of monomer sugars formed. Furthermore, Catalyst 1 was found to be more selective than Catalyst 2 at converting xylo-polysaccharides compared to gluco-polysaccharides (Table 2), where Catalyst 1 converted only $24 \%$ of the glucans contained in the corn stover (compared to $55 \%$ for Catalyst 2).

Even among a given feedstock, there can be variation in the composition. For example, two brewers' spent grain feedstocks that differ in provenance were examined. Their total carbohydrate contents differ slightly (39\% compared to $36 \%$ ), as do their compositions of gluco-polysaccharides, xylo-polysaccharides, and arabino-polysaccharides (Table 3). By varying the catalyst composition to adjust for changes in feedstock composition, the product distribution can be tuned. Fig. 6 shows the distributions of products that can be formed from these two feedstocks, using five different catalysts at the same reaction conditions. These data show that even these small differences in feedstock com- 
a)

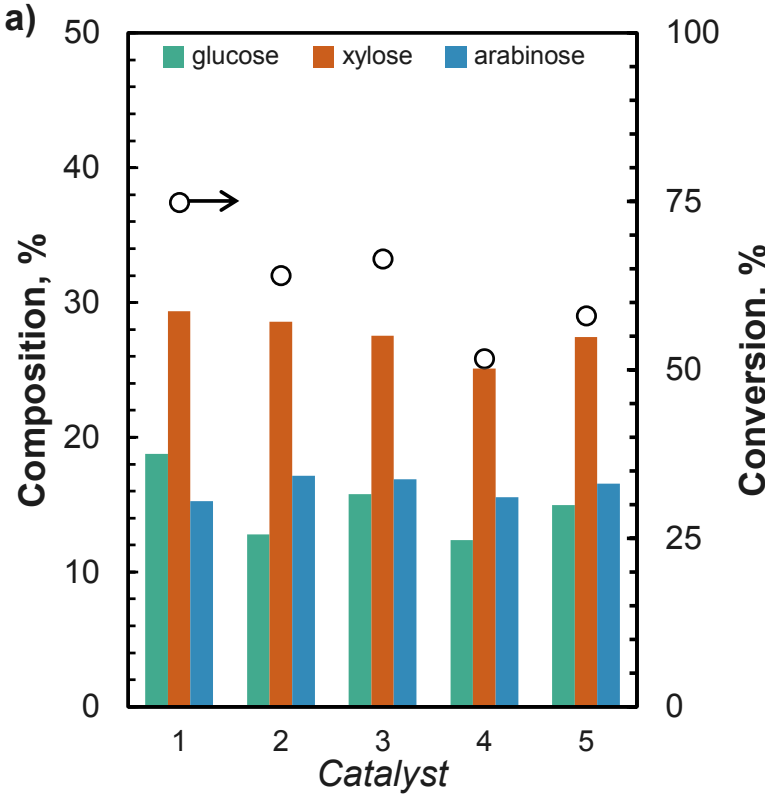

b)

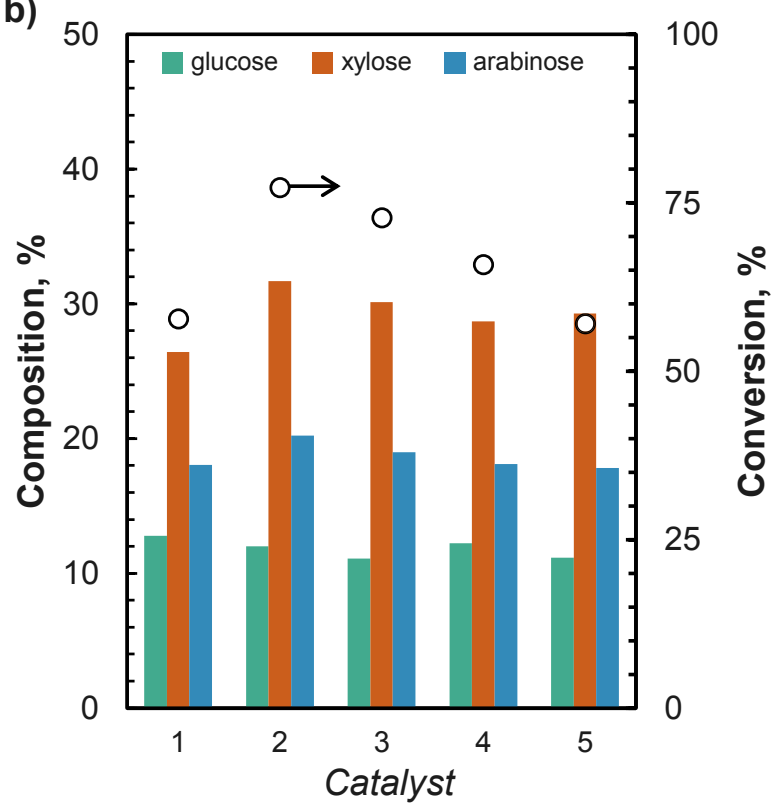

Fig. 6. Content of glucose (green), xylose (red), and arabinose (blue) in the final product and carbohydrate conversion (right axis) for five different catalysts for (a) Feedstock 1 and (b) Feedstock 2. Compositions of these feedstocks are included in Table 3.

position can result in significant variations in composition of products.

Together, these data indicate that by tuning process conditions (reaction temperature and time) and catalyst composition, biomass feedstocks can be highly utilized to obtain desired products. Our current research efforts are in expanding our library of feedstocks, catalysts, and processing parameters to develop the ability to have even more finely tuned control of product compositions. We are also investigating the selective extraction of proteins, fats, minerals, and polyphenols, among other potential products, from food and agricultural byproduct streams.

\subsection{Methods and Materials}

Catalysts were synthesized according to proprietary protocols. Biomass feedstocks were prepared by drying for $>12 \mathrm{~h}$ at $363 \mathrm{~K}$, then physically processed to obtain a smaller particle size. Catalytic tests were performed in a $300 \mathrm{~cm}^{3} 316$ stainless steel autoclave reactor (Parr). Reaction conditions are reported elsewhere, ${ }^{[22]}$ but a typical reaction is summarized here: the catalyst
$(0.1-0.5 \mathrm{~g})$ was placed within the reactor, followed by $10-50 \mathrm{~g}$ of biomass and 100-200 $\mathrm{g}$ water. The reactor was sealed and heated to reaction temperature (373-473 K), and the reaction mixture was stirred (800 rpm, using a Teflon stir bar) for the duration of the reaction $(0.5-2 \mathrm{~h})$. The reactor was cooled quickly using a cold water bath. The reaction mixture was filtered and rinsed with excess water to remove soluble products. Products were concentrated by rotary evaporation.

Two-stage acid hydrolysis was used for compositional analysis of biomass and of product oligosaccharides, following the United State Department of Energy Natural Renewable Energy Lab (NREL) laboratory analytical procedure. ${ }^{[27]}$ In short, the biomass was treated by acid hydrolysis using $1 \mathrm{~g}$ of $72 \% \mathrm{H}_{2} \mathrm{SO}_{4}$ at $303 \mathrm{~K}$ for $1 \mathrm{~h}$. The mixture was then diluted to $4 \% \mathrm{H}_{2} \mathrm{SO}_{4}$ by addition of deionized water and autoclaved for $1 \mathrm{~h}$. Sugars were analyzed using high performance liquid chromatography (Agilent 1260 Infinity) with a refractive index detector and a Hi-Plex Ca Duo ( $300 \times 7.7)$ column at $353 \mathrm{~K}$, using de-gassed distilled water as the eluent $\left(0.5 \mathrm{~cm}^{3} \mathrm{~min}^{-1}\right)$.

The degree of polymerization of oligosaccharides was characterized using mass spectrometry (Xevo ${ }^{\circledR} \mathrm{G} 2-\mathrm{S}$ QTof instrument, equipped with electrospray ionization in positive mode). The obtained spectra were centered and corrected with the lock-mass to attain $<5$ ppm mass accuracy.

\section{PREMBION ${ }^{\circledR}$ - The First in a Series of Embion Bioactives}

Based on Embion's technology platform, Embion has developed our first prebiotic offering, Prembion ${ }^{\circledR}$. Prembion is a plant-based product for the animal feed industry, manufactured from brewers's spent grain. It is a novel bioactive for microbiome nutrition and is fully water-soluble and heat and $\mathrm{pH}$ stable. It contains hydrolyzed fiber, hydrolyzed proteins, hydrolyzed lipids, and minerals, and comes in the form of a digestible powder. With its unique composition, it can represent an excellent source of nutrition for animals. From the first moments of the animal's life, Prembion provides the essential nutrients to build a strong digestive system. XOS and AXOS, derived from the fiber in brewer's spent grain, can be fermented by health-promoting bacteria and enhance production of SCFA, which are responsible for health benefits for the animal. Multiple scientific studies show that positive change in the intestinal microbiota can affect the growth performance of production animals including broilers and boost the natural mechanisms of the animal that convert feed to body weight. Bioactive peptides and amino acids confer additional biological benefits beyond their nutritional value and can enhance antimicrobial, antioxidant, antihypertensive, as well as immunomodulatory activity.

\subsection{In vitro Testing Shows Promising Prebiotic Activity}

Fig. 7 shows growth curves for a strain of Bifidobacterium that has been previously used to demonstrate efficacy of prebiotic function, grown using different carbohydrate sources. Cultures grown using Prembion ${ }^{\circledR}$ and a commercial XOS product both demonstrated exponential growth at initial times, while the control grown with glucose, did not. Surprisingly, the commercial XOS product showed a sharp decrease in cell counts after $4 \mathrm{~h}$. Cell counts remained low and increased slightly by $24 \mathrm{~h}$, with its value at $27 \mathrm{~h}$ being nearly an order of magnitude lower than the cell count for the control. In stark contrast, cell counts for Prembion $^{\circledR}$ showed continued growth to $24 \mathrm{~h}$, and after $27 \mathrm{~h}$, the cell counts have slightly decreased, indicating that the stationary growth phase was reached. These data demonstrate that Prembion ${ }^{\circledR}$ has bifidogenic properties, which indicate promise for its use as a prebiotic. 


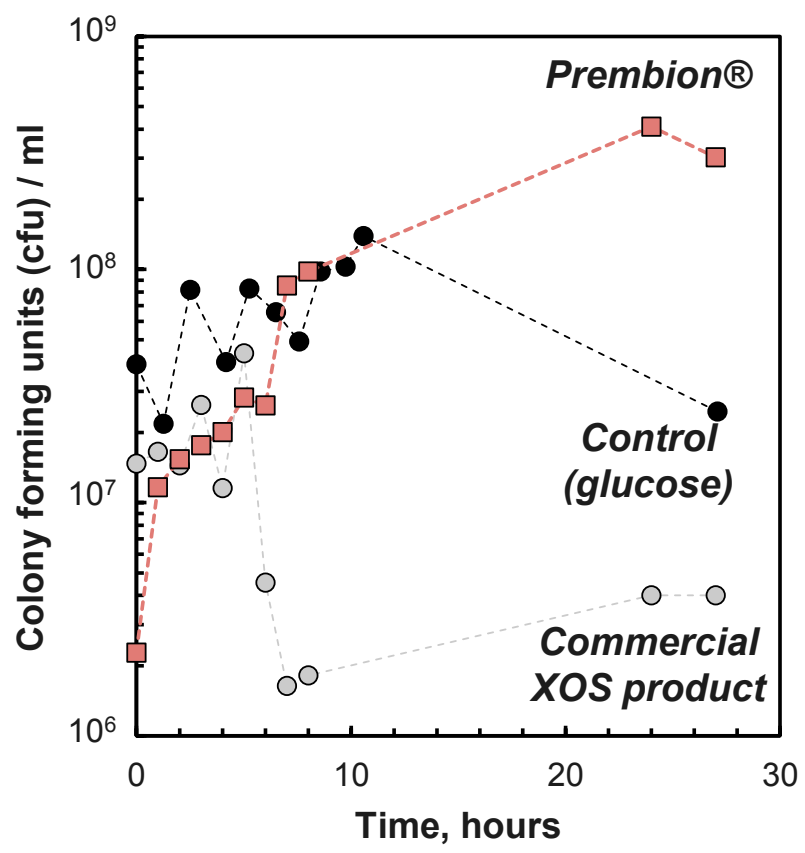

Fig. 7. Colony counts as a function of growth time for a Bifidobacterium strain that has been reported to be responsible, in part, for human and animal gut health. All additives were added on a 1\% (by total sugars basis) level.

\subsection{Methods and Materials for in vitro Testing}

The efficacy of Prembion ${ }^{\circledR}$ compared to a commercial XOS product was tested measuring the growth curves of a Bifidobacterium bacterial strain, because bifidogenic activity has been linked to improvements in animal and human gut health. The bacterial strain was pre-cultured overnight under anaerobic conditions at $37^{\circ} \mathrm{C}$ in $50 \mathrm{~mL}$ of a standard MRS broth with Tween ${ }^{\circledR}-80$ (Biolife), to which L-cysteine (Sigma) was added (0.5 g per $1000 \mathrm{~mL}$ broth).

The nutrient base medium used in fermentations was a doubly concentrated MRS broth with Tween ${ }^{\circledR}-80$, prepared without glucose (Biolife). Stock solutions (4\%) of glucose (Sigma), Prembion ${ }^{\circledR}$, and a commercial XOS product were prepared and sterilized through filtration or autoclaving. A 96 deepwell plate $(2 \mathrm{~mL}$ vol. per well; Eppendorf) was prepared by filling each well with the nutrient base medium, the carbohydrate source (to $1 \%$ carbohydrate concentration), and water in duplicate. The wells were inoculated with $20 \mu \mathrm{L}$ of the pre-culture in an anaerobic cabinet (Coy).

For cell counts, aliquots $(50 \mu \mathrm{L})$ were obtained at various times under anaerobic conditions (anaerobic cabinet, Coy). These aliquots were diluted in a sterile dilution solution $(0.85 \% \mathrm{NaCl}$, $0.1 \%$ Pepton; $1: 9$ volume ratio culture:buffer). $0.1 \mathrm{~mL}$ of each dilution was spread using a sterile L-form spreader onto sterile MRS-Agar with added L-cysteine then incubated in anaerobic conditions for $1-3$ days at $37^{\circ} \mathrm{C}$. The agar plates were visually inspected for homogeneity of colonies before these colonies were counted to allow for calculation of cell counts (colony forming units) according to ISO 7218.

\section{Outlooks}

Embion's flexible technology platform has uniquely positioned the company to enable the transition to a circular bioeconomy for a variety of food and agricultural industries. Embion's technology extracts the natural hidden value within raw materials of food and agricultural byproduct streams, including fibers, fats, proteins, minerals, and polyphenols. These extracted compounds allow us to bring different types of functional food ingredients to the food and beverage market, as well as to other markets in the future (personal care products, supplements). In addition, by targeting animal and human nutrition, Embion's process facilitates more complete utilization of arable land to improve animal and human health and immunity.

Embion's initial target is food and agricultural industry byproduct streams. However, the company's ultimate goal is to apply the technology to all producers of large waste volumes to valorize these undervalued side streams and enable the transition to a circular economy not only in Switzerland but also in Europe and the rest of the world.

\section{EMBIONTM \\ Technologies S.A.}

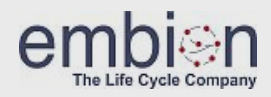

EMBIONTM Technologies S.A. is a hard-tech, disruptive spinoff of the EPFL, providing access to next-generation nutrition innovation through a novel platform technology. The patented ionic catalyst approach is based on the principle of bold simplicity - EMBION empowers a scalable and affordable way to meet the globally growing demand for plant-based nutrition of high functionality.

\section{Key Figures}

Founding Year 2016

Location(s)

Headquarter: Ecublens, Switzerland Production: Ecublens, Switzerland

Legal Form S.A.

Founders

Georgios Savoglidis Sviatlana Siankevich Georgios Fengos

Scientific Advisors Prof. Dr. Paul J. Dyson Dr. Johannes Baensch Dr. Raf Crabbe

No of employees 12

\section{Key Inventions}

Embion core ionic catalyst platform

Platform catalytic technology using a new class of proprietary, food-compatible biocatalysts for innovating across industries in the bioeconomy

Prembion ${ }^{\circledR}$

Innovative prebiotic product for animal nutrition

\section{Next Milestones}

December 2020

First ton-scale production of Prembion ${ }^{\circledR}$

January 2021

Deployment in other market segments in nutrition (protein) and in other industries (e.g., materials)

\section{Awards}

Dec. 2015

enable grant (EPFL Technology Transfer Office)

Firmenich Interal Runway invitee and presenter

2016

Upon Foundation endorsement, EPFL spin-off 


\begin{tabular}{|l|l|}
\hline 2016 & SPEI VD support \\
\hline 2016 & $\begin{array}{l}\text { Innovaud FIT seed loan } \\
\text { CTI Swiss Innovation Grant }\end{array}$ \\
\hline Oan. 2017 & $\begin{array}{l}\text { Alpine High-Tech Venture Forum: } \\
\text { Top presenter award }\end{array}$ \\
\hline Feb. 2017 & IMD Lausanne startup support \\
\hline May 2018 & Forum 5i: 1st place investor award \\
\hline May 2018 & MassChallenge Switzerland Finalist \\
\hline May 2018 & Climate-KIC Stage 1 grant \\
\hline 2019 & Innosuisse-supported company \\
\hline 2020 & Innosuisse China Camp participant \\
\hline
\end{tabular}

\section{Memberships}

Since 2017

\section{Contact}

Contact Person

\section{Sviatlana Siankevich, CTO}

Address

Embion Technologies SA

Chemin de la Dent d'Oche 1A

Building L, EPFL Innovation Park 1024-CH Ecublens

Email

Website

ssiankevich@embiontech.com

https://www.embiontech.com

\section{Acknowledgements}

The authors acknowledge Laure Menin, Daniel Ortiz Trujillo, and Francisco Sepulveda (EPFL ISIC Mass Spectrometry Platform) for mass spectrometry measurements and Prof. Dr. Paul Dyson (EPFL) for scientific discussions.

Received: June 30, 2020

[1] X. Turon, J. Venus, M. Arshadi, M. Koutinas, C. S. K. Lin, A. Koutinas, BioResources 2014, 9, 5774, DOI: 10.15376/biores.9.4.5774-5777.

[2] FAO, 'Food wastage footprint', 2013.

[3] P. Bharat Helkar, A. Sahoo, Int. J. Waste Resour. 2016, 6, DOI: 10.4172/22525211.1000248

[4] P. J. Turnbaugh, R. E. Ley, M. Hamady, C. M. Fraser-Liggett, R. Knight, J. I. Gordon, Nature 2007, 449, 804, DOI: 10.1038/nature06244.

[5] J. Lloyd-Price, G. Abu-Ali, C. Huttenhower, Genome Med. 2016, 8, 51, DOI: $10.1186 / \mathrm{s} 13073-016-0307-y$.

[6] C. L. Maynard, C. O. Elson, R. D. Hatton, C. T. Weaver, Nature 2012, 489, 231, DOI: $10.1038 /$ nature11551.

[7] B. Dalile, L. Van Oudenhove, B. Vervliet, K. Verbeke, Nat. Rev. Gastroenterol. Hepatol. 2019, 16, 461, DOI: 10.1038/s41575-019-0157-3.

[8] M. E. Sanders, D. J. Merenstein, G. Reid, G. R. Gibson, R. A. Rastall, Nat. Rev. Gastroenterol. Hepatol. 2019, 16, 605, DOI: 10.1038/s41575-019-0173-3.

[9] A. M. Mowat, W. W. Agace, Nat. Rev. Immunol. 2014, 14, 667, DOI: $10.1038 /$ nri3738

[10] E. D. Sonnenburg, S. A. Smits, M. Tikhonov, S. K. Higginbottom, N. S. Wingreen, J. L. Sonnenburg, Nature 2016, 529, 212, DOI: 10.1038/nature16504.

[11] K. E. Morrison, E. Jašarević, C. D. Howard, T. L. Bale, Microbiome 2020, 8, 15, DOI: 10.1186/s40168-020-0791-6.

[12] G. R. Gibson, R. Hutkins, M. E. Sanders, S. L. Prescott, R. A. Reimer, S. J. Salminen, K. Scott, C. Stanton, K. S. Swanson, P. D. Cani, K. Verbeke, G. Reid, Nat. Rev. Gastroenterol. Hepatol. 2017, 14, 491, DOI: 10.1038/nrgastro.2017.75.

[13] G. T. Choque Delgado, W. M. da S. C. Tamashiro, M. R. M. Junior, Y. M F. Moreno, G. M. Pastore, Food Res. Int. 2011, 44, 3167, DOI: 10.1016/j. foodres.2011.07.032.

[14] C. Amorim, S. C. Silvério, K. L. J. Prather, L. R. Rodrigues, Biotechnol. Adv. 2019, 37, 107397, DOI: 10.1016/j.biotechadv.2019.05.003.

[15] K. R. Niness, J. Nutr. 1999, 129, 1402S, DOI: 10.1093/jn/129.7.1402S.

[16] 'Prebiotics Market Analysis By Ingredient (FOS, Inulin, GOS, MOS), By Application (Food \& Beverages, Animal Feed, Dietary Supplements) And Segment Forecasts To 2024', https://www.prnewswire.com/news-releases/ prebiotics-market-analysis-by-ingredient-fos-inulin-gos-mos-by-application-food--beverages-animal-feed-dietary-supplements-and-segment-forecasts-to-2024-300352039.html, accessed June 24, 2020.

[17] A. L. Ho, O. Kosik, A. Lovegrove, D. Charalampopoulos, R. A. Rastall, Carbohydr. Polym. 2018, 179, 50, DOI: 10.1016/j.carbpol.2017.08.077.

[18] 'Prebiotics: Development \& Application', Eds. G. Gibson, R. Rastall, John Wiley \& Sons, West Sussex, England, 2006.

[19] W. F. Broekaert, C. M. Courtin, K. Verbeke, T. Van de Wiele, W Verstraete, J. A. Delcour, Crit. Rev. Food Sci. Nutr. 2011, 51, 178, DOI: 10.1080/10408390903044768.

[20] M. H. Kogut, Anim. Feed Sci. Technol. 2019, 250, 32, DOI: 10.1016/j.anifeedsci.2018.10.008

[21] N. K. Morgan, C. Keerqin, A. Wallace, S.-B. Wu, M. Choct, Anim. Nutr 2019, 5, 56, DOI: 10.1016/j.aninu.2018.05.001.

[22] P. Dyson, Z. Fei, S. Siankevich, G. Savoglidis, US 2019/0031797 A1, 2019.

[23] P. Walden, Извьстія Императорской Академіи Наукъ. VI серія 1914, 8 , 405.

[24] R. P. Swatloski, S. K. Spear, J. D. Holbrey, R. D. Rogers, J. Am. Chem. Soc. 2002, 124, 4974, DOI: 10.1021/ja025790m.

[25] H. Wang, G. Gurau, R. D. Rogers, Chem. Soc. Rev. 2012, 41, 1519, DOI: $10.1039 / \mathrm{c} 2 \mathrm{cs} 15311 \mathrm{~d}$.

[26] I. Krossing, J. M. Slattery, C. Daguenet, P. J. Dyson, A. Oleinikova, H Weingärtner, J. Am. Chem. Soc. 2006, 128, 13427, DOI: 10.1021/ja0619612.

[27] A. Sluiter, B. Hames, R. Ruiz, C. Scarlata, J. Sluiter, D. Templeton, D. Crocker, Lab. Anal. Proced. 2012, 17, DOI: NREL/TP-510-42618.

\section{License and Terms}

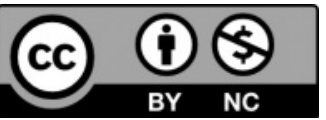

This is an Open Access article under the terms of the Creative Commons Attribution License CC BY_NC 4.0. The material may not be used for commercial purposes.

The license is subject to the CHIMIA terms and conditions: (http:// chimia.ch/component/sppagebuilder/?view=page \&id=12).

The definitive version of this article is the electronic one that can be found at https://doi.org/10.2533/chimia.2020.784 